\title{
Correction to: Teaching, Affirming, and Recognizing Trans and Gender Creative Youth
}

\author{
sj Miller
}

\section{Correction to:}

sj Miller (ed.), Teaching, Affirming, and Recognizing Trans and Gender Creative Youth, https://doi.org/10.1057/978-1-137-56766-6

The following changes are done in the book.

1) The affiliation of all 3 series editors' are updated.

2) International Advisory Board details are updated in the series information text page.

3) The volume editor affiliation is updated.

The updated original online version of the book can be found at https://doi.org/10.1057/978-1-137-56766-6

(C) The Author(s) 2019 